Ying Guo and Ban-Feng Ruan*

\title{
The crystal structure of (4-nitrophenyl) (5-ferrocenyl-3-(trifluoromethyl)-1H-pyrazol-1-yl) methanone, $\mathrm{C}_{21} \mathrm{H}_{12} \mathrm{~F}_{3} \mathrm{FeN}_{3} \mathrm{O}_{3}$
}

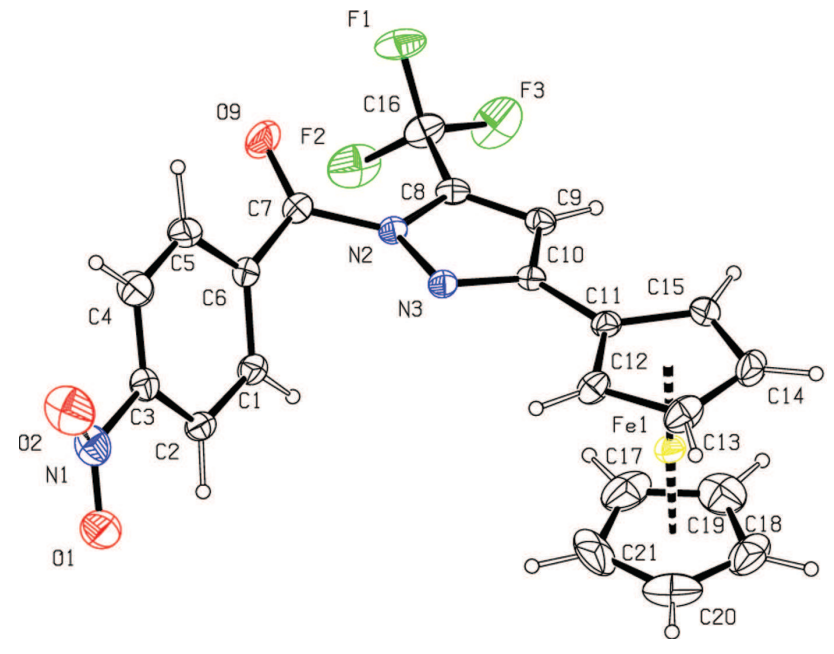

https://doi.org/10.1515/ncrs-2018-0102

Received March 10, 2018; accepted July 16, 2018; available online July 31, 2018

\begin{abstract}
$\mathrm{C}_{21} \mathrm{H}_{14} \mathrm{~F}_{3} \mathrm{FeN}_{3} \mathrm{O}_{3}$, triclinic, $P \overline{1} \quad$ (no. 2), $\quad a=7.961$ (3) $\AA$, $b=11.136(4) \AA, \quad c=11.588(4) \AA, \quad \alpha=76.357(10)^{\circ}$, $\beta=84.998(10)^{\circ}, \quad \gamma=76.989(10)^{\circ} \quad V=972.1(6) \AA^{3}, \quad Z=2$, $R_{\mathrm{gt}}(F)=0.0509, w R_{\text {ref }}\left(F^{2}\right)=0.1304, T=273(2) \mathrm{K}$.
\end{abstract}

CCDC no.: 1856034

The crystal structure is shown in the figure. Tables 1 and 2 contain details on crystal structure and measurement conditions and a list of the atoms including atomic coordinates and displacement parameters.

\section{Source of materials}

A mixture of 5-ferrocenyl-3-(trifluoromethyl)- $1 H$-pyrazole (0.32 g, $1 \mathrm{mmol}$, prepared by a literature method [4]), triethylamine $(0.17 \mathrm{~mL}, 1.2 \mathrm{mmol})$, and 4-nitrobenzoyl

\footnotetext{
*Corresponding author: Ban-Feng Ruan, School of Biological and Medical Engineering, Hefei University of Technology, Hefei 230009, People's Repulic of China, e-mail: ruanbf@hfut.edu

Ying Guo: Department of Biology, Chemistry and Environmental Engineering, Haijiang Normal University, Shiyan 442500, People's Repulic of China
}

Table 1: Data collection and handling.

\begin{tabular}{ll}
\hline Crystal: & Clear dark red block \\
Size: & $0.25 \times 0.23 \times 0.21 \mathrm{~mm}$ \\
Wavelength: & Mo $K \alpha$ radiation $(0.71073 \AA$ A $)$ \\
$\mu:$ & $0.83 \mathrm{~mm}^{-1}$ \\
Diffractometer, scan mode: & SMART, $\varphi$ and $\omega$-scans \\
$\theta_{\text {max }}$, completeness: & $26^{\circ},>99 \%$ \\
$N(h k l)_{\text {measured }}, N(h k l)_{\text {unique }}, R_{\text {int }}:$ & $9817,3821,0.039$ \\
Criterion for $I_{\text {obs }}, N(h k l)_{\text {gt }}:$ & $I_{\text {obs }}>2 \sigma\left(I_{\text {obs }}\right), 2643$ \\
$N(\text { param })_{\text {refined }}:$ & 289 \\
Programs: & Bruker programs [1], SHELX [2, 3] \\
\hline
\end{tabular}

chloride $(0.22 \mathrm{~g}, 0.012 \mathrm{~mol})$ in $5 \mathrm{~mL}$ of $\mathrm{CH}_{2} \mathrm{Cl}_{2}$ were refluxed overnight. After completion, the solvent was evaporated under reduced pressure. Water was added and the mixture was extracted with ethyl acetate twice. The combined organic layer was dried over $\mathrm{Na}_{2} \mathrm{SO}_{4}$, filtered and evaporated. The pure title compound was obtained by column chromatography on silica gel using petroleum ether/ethylacetate (2:1, $v / v, \operatorname{Rf}=0.2)$ as eluent. Crystals of the title compound were obtained by slow evaporation of dichloromethane/methanol within 2 days.

\section{Experimental details}

Single crystal X-ray diffraction measurements for title compound were carried out on a Siemens Smart 1000 CCD diffractometer equipped with a graphite crystal monochromator situated in the incident beam for data collection at room temperature. The hydrogen atoms were added theoretically and riding on the concerned atoms.

\section{Comment}

Organometallic compounds, which are defined as metal complexes containing at least one direct metal-carbon bond, have been proven to be a promising metal-based chemotherapy alternative option to platinum-based anticancer drugs $[5,6]$. Among them, the use of ferrocene-containing compounds for medicinal applications has long been considered as an attractive way to develop anticancer drugs [7, 8], due to its low toxicity, significant stability and lipophilicity, facile functionalization, and unique electrochemical behavior. In the past few years, we have reported several ferrocenes and 
Table 2: Fractional atomic coordinates and isotropic or equivalent isotropic displacement parameters $\left(\AA^{2}\right)$.

\begin{tabular}{|c|c|c|c|c|}
\hline Atom & $x$ & $y$ & $z$ & $U_{\text {iso }} * / U_{\text {eq }}$ \\
\hline $\mathrm{Fe} 1$ & $0.75072(6)$ & $-0.22990(4)$ & $0.96441(4)$ & $0.04639(19)$ \\
\hline $\mathrm{F} 1$ & $1.3456(3)$ & $0.1125(2)$ & $0.5665(2)$ & $0.0862(8)$ \\
\hline F2 & $1.1666(4)$ & $0.0785(3)$ & $0.4588(2)$ & $0.1040(9)$ \\
\hline F3 & $1.3307(4)$ & $-0.0758(3)$ & $0.5675(3)$ & $0.1087(10)$ \\
\hline 01 & $0.1424(4)$ & $0.5165(3)$ & $0.6849(3)$ & $0.0876(10)$ \\
\hline $02^{\mathrm{a}}$ & $0.264(2)$ & $0.6429(13)$ & $0.7359(11)$ & $0.108(4)$ \\
\hline $02^{\text {b }}$ & $0.2614(19)$ & $0.6768(11)$ & $0.6659(9)$ & $0.092(3)$ \\
\hline 09 & $1.0422(3)$ & $0.2998(3)$ & $0.5523(3)$ & $0.0827(9)$ \\
\hline N1 & $0.2687(5)$ & $0.5589(3)$ & $0.6836(3)$ & $0.0686(9)$ \\
\hline $\mathrm{N} 2$ & $0.9667(3)$ & $0.1290(2)$ & $0.6769(2)$ & $0.0399(6)$ \\
\hline N3 & $0.8720(3)$ & $0.0912(2)$ & $0.7788(2)$ & $0.0411(6)$ \\
\hline $\mathrm{C} 1$ & $0.6087(4)$ & $0.2869(3)$ & $0.6338(3)$ & $0.0483(8)$ \\
\hline $\mathrm{H} 1$ & 0.617451 & 0.204733 & 0.625071 & $0.058^{\star}$ \\
\hline $\mathrm{C} 2$ & $0.4488(4)$ & $0.3621(3)$ & $0.6465(3)$ & $0.0477(8)$ \\
\hline $\mathrm{H} 2$ & 0.348908 & 0.332750 & 0.643911 & $0.057^{\star}$ \\
\hline $\mathrm{C} 3$ & $0.4399(4)$ & $0.4808(3)$ & $0.6630(3)$ & $0.0477(8)$ \\
\hline $\mathrm{C} 4$ & $0.5833(5)$ & $0.5294(4)$ & $0.6620(4)$ & $0.0661(11)$ \\
\hline $\mathrm{H} 4$ & 0.572944 & 0.611067 & 0.672386 & 0.079 * \\
\hline $\mathrm{C} 5$ & $0.7424(5)$ & $0.4560(3)$ & $0.6453(4)$ & $0.0634(10)$ \\
\hline $\mathrm{H} 5$ & 0.840915 & 0.488663 & 744 & $0.076^{*}$ \\
\hline $\mathrm{C} 6$ & $0.7568(4)$ & $0.3324(3)$ & $0.6338(3)$ & $0.0448(8)$ \\
\hline $\mathrm{C} 7$ & $0.9316(4)$ & $0.2570(3)$ & $0.6154(3)$ & $0.0507(9)$ \\
\hline $\mathrm{C} 8$ & $1.1069(4)$ & $0.0343(3)$ & $0.6640(3)$ & $0.0425(8)$ \\
\hline C9 & $1.1003(4)$ & $-0.0643(3)$ & $0.7564(3)$ & $0.0413(7)$ \\
\hline H9 & 1.176687 & -0.142365 & 0.771058 & $0.050^{*}$ \\
\hline C10 & $0.9535(4)$ & $-0.0245(3)$ & $0.8261(3)$ & $0.0365(7)$ \\
\hline C11 & $0.8928(4)$ & $-0.0946(3)$ & $0.9398(3)$ & $0.0415(7)$ \\
\hline $\mathrm{C} 12$ & $0.7364(5)$ & $-0.0569(3)$ & $1.0010(3)$ & $0.0515(9)$ \\
\hline $\mathrm{H} 12$ & 0.652661 & 0.015783 & 0.975804 & $0.062^{\star}$ \\
\hline $\mathrm{C} 13$ & $0.7288(6)$ & $-0.1493(4)$ & $1.1079(3)$ & $0.0656(11)$ \\
\hline $\mathrm{H} 13$ & 0.639393 & -0.147441 & 149 & 0.079 * \\
\hline C14 & $0.8781(6)$ & $-0.2431(4)$ & $1.1124(3)$ & $0.0658(11)$ \\
\hline $\mathrm{H} 14$ & 0.904928 & -0.315042 & 1.173094 & 0.079 * \\
\hline C15 & $0.9823(5)$ & $-0.2109(3)$ & $1.0094(3)$ & $0.0544(9)$ \\
\hline H15 & 1.089553 & -0.257254 & 0.990584 & $0.065^{\star}$ \\
\hline C16 & $1.2368(5)$ & $0.0397(4)$ & $0.5640(4)$ & $0.0616(10)$ \\
\hline $\mathrm{C} 17$ & $0.7348(9)$ & $-0.2717(6)$ & $0.8063(4)$ & $0.1003(16)$ \\
\hline $\mathrm{H} 17$ & 0.794942 & -0.240812 & 0.737277 & 0.120 * \\
\hline C18 & $0.7882(7)$ & $-0.3812(5)$ & $0.8882(5)$ & $0.0932(15)$ \\
\hline $\mathrm{H} 18$ & 0.891723 & -0.439025 & 0.884038 & $0.112^{*}$ \\
\hline C19 & $0.6655(8)$ & $-0.3918(5)$ & $0.9765(5)$ & $0.0942(15)$ \\
\hline $\mathrm{H} 19$ & 0.671269 & -0.458675 & 1.042634 & $0.113^{*}$ \\
\hline $\mathrm{C} 20$ & $0.5345(7)$ & $-0.2909(7)$ & $0.9538(6)$ & $0.1121(18)$ \\
\hline $\mathrm{H} 2 \mathrm{O}$ & 0.435555 & -0.274557 & 1.001413 & $0.134^{*}$ \\
\hline $\mathrm{C} 21$ & $0.5745(9)$ & $-0.2155(6)$ & $0.8457(7)$ & $0.124(2)$ \\
\hline $\mathrm{H} 21$ & 0.505339 & -0.140321 & 0.806701 & $0.149 *$ \\
\hline
\end{tabular}

Occupancies: ${ }^{\mathrm{a}}=0.48,{ }^{\mathrm{b}}=0.52$

found that some of them displayed impressive anticancer activities [9, 10]. Herein we report the synthesis and crystal structure of a ferrocene-based amide bearing a pyrazolyl moiety.
The title compound crystallizes in the triclinic space group $P \overline{1}$, with two molecules in the unit cell. The average bond length of Fe1-C (Cp11-15) is $2.036 \AA$, which is comparable to that (2.02 $\AA$ ) of the other $\mathrm{Cp}$ ring (Cp17-21). The longest bond length is Fe1 … C13 (2.045 $\AA$ ), and the shortest Fe-C (Cp) separation is $2.02 \AA$ (Fe1 ‥ C21). The Cp-Fe-Cp angle (179.3) deviates slightly from linearity. The pyrazole moiety is rotated out the plane of the adjacent $\mathrm{Cp}$ ring (C17-C18-C19-C20-C21) by approximately $8.9^{\circ}$.

Acknowledgements: This work was supported by the Key Scientific and Technological Project of Anhui Provincial Tobacoo Company (No. 20150551007).

\section{References}

1. Bruker: APEX3, SAINT-Plus, XPREP. Bruker AXS Inc., Madison, WI, USA (2016).

2. Sheldrick, G. M.: SHELXT - Integrated space-group and crystal-structure determination. Acta Crystallogr. A71 (2015) 3-8.

3. Sheldrick, G. M.: Crystal structure refinement with SHELXL. Acta Crystallogr. C71 (2015) 3-8.

4. Huang, X.-F.; Tang, J.-F.; Ji, J.-L.; Wang, X.-L.; Ruan, B.-F.: Synthesis, characterization and antitumor activity of novel amide derivatives containing ferrocenyl pyrazol-moiety. J. Organomet. Chem. 706 (2012) 113-123.

5. Zhang, P.; Sadler, P. J.: Advances in the design of organometallic anticancer complexes. J. Organomet. Chem. 839 (2017) 5-14.

6. Nieto, D.; Bruña, S.; González-Vadillo, A. M.; Perles, J.; Carrillo-Hermosilla, F.; Antiñolo, A.; Padrón, J. M.; Plata, G. B.; Cuadrado, I.: Catalytically generated ferrocene-containing guanidines as efficient precursors for new redox-active heterometallic platinum(II) complexes with anticancer activity. Organometallics. 34 (2015) 5407-5417.

7. Chopra, P.; de Kock, C.; Smith, P.; Chibale, K.; Singh, K.: Ferrocene-pyrimidine conjugates: synthesis, electrochemistry, physicochemical properties and antiplasmodial activities. Eur. J. Med. Chem. 100 (2015) 1-9.

8. Braga, S. S.; Silver, A. M. S.: A new age for iron: antitumoral ferrocenes. 32 (2013) 5626-5639.

9. Huang, X.-F.; Wang, L.-Z.; Tang, L.; Lu, Y.-X.; Wang, F.; Song, G.-Q.; Ruan, B.-F.: Synthesis, characterization and antitumor activity of novel ferrocene derivatives containing pyrazolyl-moiety. J. Organomet. Chem. 749 (2014) 157-162.

10. Ren, J.; Wang, S.-Q.; Ni, H.-J.; Yao, R.-S.; Liao, C.-Z.; Ruan, B.-F.: Synthesis, characterization and antitumor activity of novel ferrocene-based amides bearing pyrazolyl moiety. J. Inorg. Organomet. Polym. 25 (2015) 419-426. 